\title{
Correlação da evolução do estado funcional com o tempo de ventilação mecânica invasiva em pacientes críticos
}

\section{Correlation of functional status evolution with invasive mechanical ventilation time in critically ill patients}

\author{
(1D) Marilucia Reis dos Santos ${ }^{1}$, Caroline Ferreira Guerreiro ${ }^{1}$, Jorge Luis Motta Anjos ${ }^{1}$, Cássio Magalhães da
} Silva e Silva ${ }^{2}$

\begin{abstract}
RESUMO
Pacientes críticos internados na unidade de terapia intensiva (UTI) comumente apresentam declínio do estado funcional, sendo o tempo de permanência na ventilação mecânica invasiva (VMI) o fator de maior importância relacionado ao comprometimento do desempenho físico. Objetivo: Investigar a correlação da evolução do estado funcional entre a admissão e a alta com o tempo de VMI relacionada a mobilidade. Método: Trata-se de um estudo observacional, de delineamento transversal, realizado nas UTI's de um hospital da rede pública estadual. Foi aplicado a escala Functional Status Score Intensive Unit Care (FSS-ICU) por fisioterapeutas treinados para avaliação do estado funcional. Para comparação os grupos foram divididos em clínicos e cirúrgicos e utilizado o teste não paramétrico de Mann-Whitney. Para análise da correlação foi utilizado o coeficiente de Spearman. Resultados: A amostra foi composta por 30 pacientes com mediana de idade 49,5 [37,0-67,7] anos, sendo a maioria (53\%) do sexo masculino, com mediana de tempo de VMI 132,0 [48,0-192,0] horas, mediana de 4,0 [2,0-6,0] do índice de comorbidades de Charlson, predomínio das cirurgias abdominais (75\%) e mediana do FSS entre a admissão e a alta de 12, 0 [10,0-30,7]. Verificou-se uma correlação negativa de moderada a boa entre o FSS da admissão e da alta com o tempo de VMI em horas $(r=-0,50 p=$ $0,005)(r=-0,71 p<0,001)$. Conclusão: Existe correlação negativa entre a evolução do estado funcional na admissão e alta com o tempo de VMI em doentes críticos e o perfil diagnóstico também pode interferir nesse desfecho.
\end{abstract}

Palavras-chave: Respiração Artificial, Cuidados Críticos, Atividades Diárias, Limitação da Mobilidade

\begin{abstract}
Intensive care patients admitted to the intensive care unit (ICU) usually present a decline in functional status, and the length of stay in invasive mechanical ventilation (IMV) is the most important factor related to impaired physical performance. Objective: To investigate the correlation of the evolution of the functional state between the admission and the rise with the time of VMI made a list of the mobility. Method: This is an observational, cross-sectional study, performed at the ICUs of a state public hospital. Functional Status Score Intensive Care Unit (FSS-ICU) was applied by physiotherapists trained to evaluate functional status. For comparison the groups were divided in clinically and surgically and when the test was used not parametric of Mann-Whitney. The Spearman coefficient was used to analyze the correlation. Results: The sample consisted of 30 patients, 53\% male, with median age 49.50 [37.0-67.7] years, median time to IMV 132.00 [48.0-192.0] hours, Charlson comorbidities index 4.0 [2.0-6.0], predominance of the abdominal surgeries ( $75 \%$ ) and median of the FSS between the admission and the high of $12,0[10,0-30,7]$. There was a moderate to good negative correlation between the FSS of the admission and of the high with the time of VMI in hours $(r=-0,50 p=0,005)(r=-$ $0,71 p<0,001)$. Conclusion: There is a negative correlation between the evolution of the functional state in the admission and high with the time of VMI in critical patients and the diagnostic profile also can interfere in this ending.
\end{abstract}

Keywords: Respiration, Artificial, Critical Care, Activities of Daily Living, Mobility Limitation 


\section{INTRODUÇÃO}

A unidade de terapia intensiva (UTI) utiliza recursos humanos e materiais para o acolhimento de pacientes em estado grave, potencialmente recuperáveis, que requerem monitoramento constante e suporte avançado de vida por uma equipe especializada. ${ }^{1,2,3}$ Contudo, apesar de todos os avanços científicos e tecnológicos, possui fatores que contribuem para redução do desempenho físico como uso de sedativos e drogas vasoativas, dispositivos invasivos, restrição ao leito e ventilação mecânica invasiva (VMI). $4,5,6$

Os pacientes admitidos na UTI comumente apresentam declínio do estado funcional decorrente do tempo prolongado de uso da sedação, instabilidade clínica, presença de comorbidades e condições nutricionais associadas ao doente crítico. ${ }^{7,8}$ Entretanto, os fatores de maior importância relacionado ao comprometimento do desempenho físico são o tempo de permanência na VMI e o tempo de internamento hospitalar. ${ }^{9,10}$

O suporte ventilatório invasivo é utilizado por $39 \%$ dos pacientes internados em UTI, sendo que $10 \%$ necessitam de ventilação mecânica prolongada, o que contribui para a redução de 25 a $60 \%$ da força muscular periférica com repercussões em transferências e marcha. ${ }^{10}$ Além disso, seu uso prolongado está associado a complicações cardiopulmonares, alterações cognitivas e na qualidade de vida, que podem permanecer até cinco anos após alta, comprometendo a vida social do indivíduo. ${ }^{11,12}$

A utilização de ferramentas para caracterização da capacidade funcional na UTI tem relevância na predição de desfechos negativos como sarcopeniae quedas, sinalizando para utilização de tratamentos específicos. O Functional Status Score Intensive Unit Care (FSS-ICU) é considerado como um instrumento de fácil compreensão e aplicabilidade clínica para avaliação do estado funcional nesse ambiente especifico. ${ }^{6,13}$

Apesar de existirem na literatura estudos que avaliem o impacto do internamento hospitalar nas transferências e atividades básicas de vida diária (AVD), poucos correlacionaram o tempo de VMI com a capacidade funcional no doente crítico utilizando-se de escalas padronizadas e com propriedades psicométricas adequadas.

\section{OBJETIVO}

Investigar a correlação da evolução do estado funcional entre a admissão e a alta com o tempo de VMI relacionada a mobilidade.

\section{MÉTODOS}

Trata-se de um estudo observacional, de delineamento transversal, realizado nas unidades de terapia intensiva de um hospital da rede pública estadual, localizado na cidade de Salvador-BA, no período de dezembro de 2017 a novembro de 2018.

A pesquisa é parte integrante de um projeto maior intitulado: Avaliação da funcionalidade em pacientes internados em um hospital de alta complexidade de Salvador-BA.

Foram incluídos no estudo indivíduos com idade superior a 18 anos e em uso de VMI, sendo excluídos aqueles com instabilidade hemodinâmica, doenças arteriais obstrutivas periféricas e/ou amputação de membros inferiores, presença de sequelas neurológicas e uso de sedação com Escala de Sedação e Agitação de Richmond (RASS) <-2 (sedação de moderada a profunda) ou > +1 (agitado).

A variável primária mensurada na amostra foi o estado funcional utilizando-se a escala Functional Status Score Intensive Unit Care (FSSICU).

Trata-se de uma escala validadae adaptada culturalmente para o português do Brasil. Avalia a realização de cinco tarefas, com uma pontuação total que varia de 0 a 35 pontos, sendo atribuído uma numeração que varia de zero (totalmente incapaz de realizar) até 7 (independência completa) para cada atividade. ${ }^{14,15}$
No estudo foi contabilizado tanto a pontuação total do FSS, quanto as categorias, sendo atribuído uma ordem alfabética para cada atividade avaliada: A (rolar), B (transferência da posição supina para sentada), C (transferência da posição sentada para em pé), D (sentarse a beira leito) e $E$ (deambulação). Nenhum paciente deambulou em uso de VMI, sendo atribuído pontuação zero na admissão.

Os dados secundários foram extraídos dos prontuários como idade, sexo, data de admissão no hospital e na UTI, motivo da internação com diagnóstico a partir do prontuário médico, sedação, tipo de via aérea, dados de ventilação mecânica como data de intubação traqueal extubação, tempo de VMI e de internamento na UTI, desfecho e o Índice Comorbidadesde Charlson, que avalia a carga de morbidade dos doentes e prediz mortalidade. Sendo composto por uma lista de condições clínicas secundárias, para as quais sãoatribuídos pesos que variam de zero a seis, que somados geram um escore final de gravidade. ${ }^{16,17}$

Vale salientar que as UTIs tinham assistência fisioterapêutica durante 24 horas por dia com foco na mobilização precoce respeitando os critérios de segurança preconizados na literatura. ${ }^{13}$

As condutas de mobilização eram realizadas por dois períodos diariamente, com duração de 20 a 40 minutos a depender da gravidade dos pacientes. Os atendimentos realizados incluíam cinesioterapia global, treino de transferências, sedestação com membros inferiores apoiados, ortostase e treino de marcha.

A aplicação das escalas foi realizada por fisioterapeutas capacitados, diretamente ao paciente, após instrução e treinamento prévio com padronização dos métodos. Os pacientes foram orientados e posteriormente foram dados os comandos para a realização das tarefas, sempre com a assistência de dois profissionais, seja na realização do movimento ou no cuidado com o circuito do ventilador mecânico para evitar possíveis intercorrências.

A deambulação foi avaliada a beira leito, com o deslocamento do ventilador até um limite possível para realização da marcha. Em seguida, o paciente era reposicionado de maneira confortável no leito, devidamente monitorizado.

Para análise dos dados demográficos e clínicos, foram utilizadas estatísticas descritivas. Os dados de variáveis contínuas foram avaliados com medidas de tendência central e dispersão e expressos como medias, medianas, desvio-padrão e intervalos de confiança. Dados de variáveis dicotômicas ou categóricas foram avaliados com medidas de frequência e expressos como porcentagens, com o uso do software SPSS (StatisticalPackage for the Social Sciences) for Windows (versão 22.0).

Para comparação os grupos foram divididos em clínicos e cirúrgicos de acordo com o diagnóstico médico, sendo avaliado inicialmente a normalidade da distribuição e após utilizado o teste não paramétrico de Mann-Whitney.

Esses dados foram apresentados através de tabelas e gráficos. 0 nível de confiança adotado foi de $95 \%$, considerando um valor de $p<$ 0,05 para se obter significância estatística. Para medir a correlação das variáveis explicativas sobre o desfecho primário, foi utilizado teste de correlação de Spearman.

Para análise dos resultados, coeficientes entre 0,00 a 0,25 foram considerados indicando pouca ou nenhuma correlação; aqueles entre 0,25 a 0,50 indicaram um grau fraco de correlação; valores entre 0,50 e 0,75 indicaram uma correlação de moderada a boa; e valores acima de 0,75 foram considerados de boa a excelente correlação. ${ }^{18} \mathrm{O}$ projeto foi aprovado pelo Comitê de Ética e Pesquisa sob protocolo 2.382.343 e CAAE 59587416.0.0000.5028. O termo de consentimento livre e esclarecido (TCLE) foi assinado pelos pacientes ou familiares.

\section{RESULTADOS}

A amostra foi composta por 30 pacientes com mediana de idade $49,5[37,0-67,7]$ anos, sendo a maioria $(53 \%)$ do sexo masculino, com mediana de tempo de VMI 132,0 [48,0-192,0] horas, mediana de 4,0 $[2,0-6,0]$ do índice de comorbidades de Charlson e mediana do FSS 
entre a admissão e a alta de 12, 0 [10,0-30,7]. Em relação aos motivos de internação houve predomínio das cirurgias abdominais (75\%), seguidos dos motivos: sepse (42\%), insuficiência cardíaca (21\%), insuficiência renal crônica (21\%) e doença parenquimatosa do fígado descompensada (14\%) (Tabela1).

Tabela 1. Características sociodemográficas e funcionais da amostra $(n=30)$

\begin{tabular}{|c|c|c|c|}
\hline Variáveis & Mediana [IQ] & n (\%) & Valor de $p$ \\
\hline Idade (anos) & $49.5[37.0-67.7]$ & & \\
\hline Tempo de VMI (horas) & $132.0[48.0-192.0]$ & & \\
\hline Sedados & & $11(36 \%)$ & \\
\hline Não sedados & & $19(63 \%)$ & \\
\hline Índice de Charlson & $4.0[2.0-6.0]$ & & \\
\hline \multicolumn{4}{|l|}{ Sexo } \\
\hline Masculino & & $16(53 \%)$ & \\
\hline Feminino & & $14(46 \%)$ & \\
\hline \multicolumn{4}{|l|}{ Perfil admissional } \\
\hline Clínico & & $14(46 \%)$ & \\
\hline Cirúrgico & & $16(53 \%)$ & \\
\hline \multicolumn{4}{|l|}{ Motivo da internação } \\
\hline Cirurgias abdominais & & $12(75 \%)$ & \\
\hline Sepse & & $6(42 \%)$ & \\
\hline Insuficiência Cardíaca & & $3(21 \%)$ & \\
\hline Insuficiência renal crônic & & $3(21 \%)$ & \\
\hline Doença crônica parenqui & atosa do fígado & $2(14 \%)$ & \\
\hline \multicolumn{4}{|c|}{ Estado Funcional } \\
\hline FSS admissão x FSS alta & $12.0[10.0-30.7]$ & & $<0.001$ \\
\hline
\end{tabular}

Teste de Mann-Whitney; IQ - intervalo interquartil; VMI - Ventilação mecânica invasiva FSS - Functional Status Score Intensive Unit Care

Na análise do estado funcional na admissão e na alta com o tempo de VMI, foi possível encontrar correlação negativa de moderada a boa entre o FSS da admissão e da alta com o tempo de VMI em horas. Vale salientar também, que não foi encontrado correlação entre o índice de comorbidades de Charlson e o tempo de VMI em horas (Tabela 2) (Figuras 1 e 2).

Tabela 2. Correlação entre estado funcional na admissão, na alta, o Índice de comorbidades de Charlson e o tempo de ventilação mecânica invasiva $(n=30)$

\begin{tabular}{lcc}
\hline \multicolumn{1}{c}{ Variáveis } & \multicolumn{2}{c}{ Tempo de VMI } \\
& $\mathbf{R}$ & $\mathbf{p}$-valor \\
\hline FSS admissão & -0.50 & $\mathrm{p}=0.006$ \\
FSS alta & -0.71 & $\mathrm{p}<0.001$ \\
Índice de Charlson & 0.34 & $\mathrm{p}=0.064$ \\
\hline
\end{tabular}

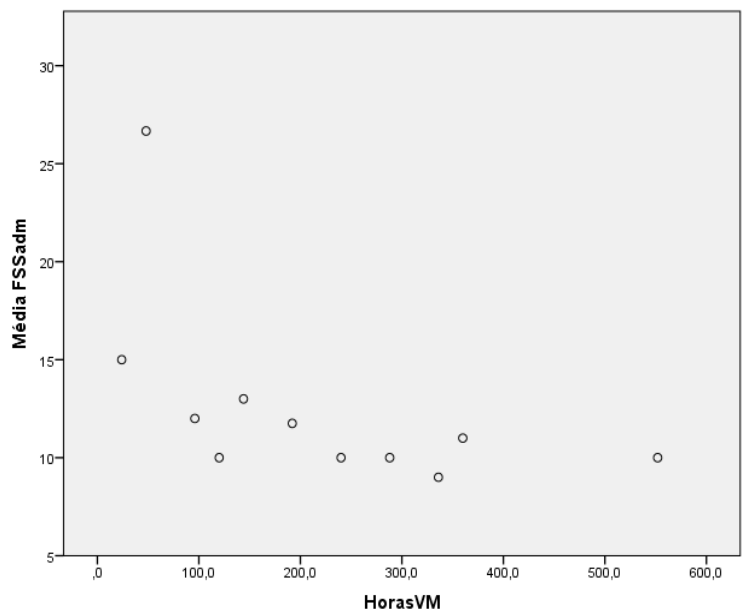

Figura 1. Correlação entre FSS da admissão e tempo de VMI em horas $(r=-0,50 p=0,005)$

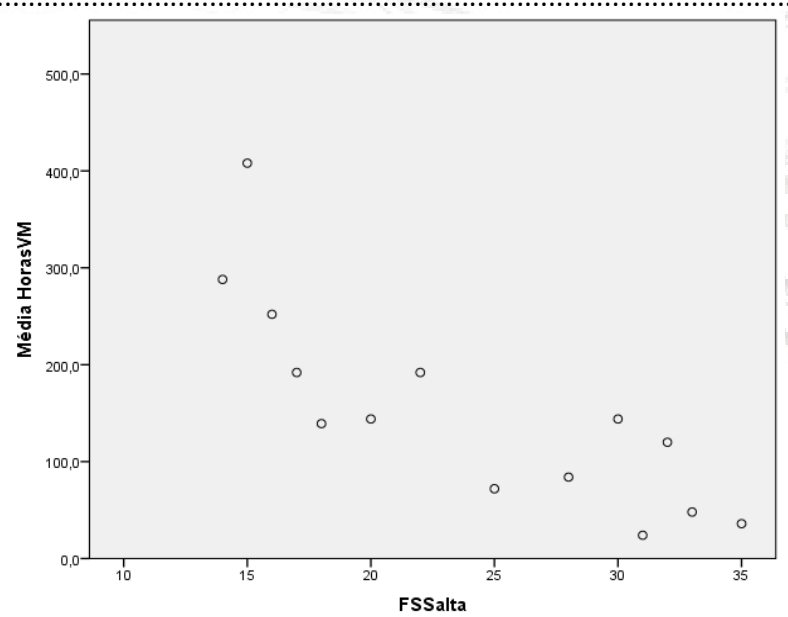

Figura 2. Correlação entre o tempo de VMI em horas e o FSS da alta $(r=-0,71 p<0,001)$

Na análise do FSS por categorias, comparando-se respectivamente cada categoria na admissão e na alta pode-se observar que houve diferença em todos os domínios $A, B, C, D$ e $E(p<0,001)$. Quando avaliado os perfis diagnósticos, não foi encontrado diferenças entre os pacientes clínicos e cirúrgicos no que diz respeito ao tempo de VMI, tempo de internação hospitalar e estado funcional na admissão, porém esta foi encontrada quando comparado o estado funcional na alta (Tabela 3).

Tabela 3. Comparação entre o estado funcional na admissão, na alta, o tempo de ventilação mecânica invasiva e o tempo de internação hospitalar entre os pacientes clínicos e cirúrgicos $(n=30)$

\begin{tabular}{llcc}
\hline \multicolumn{1}{c}{ Variáveis } & \multicolumn{1}{c}{$\begin{array}{c}\text { Clínico (n=14) } \\
\text { Mediana (IQ) }\end{array}$} & $\begin{array}{c}\text { Cirúrgico }(\mathbf{n}=16) \\
\text { Mediana (IQ) }\end{array}$ & $\begin{array}{c}\mathbf{p}- \\
\text { valor }\end{array}$ \\
\hline FSS admissão & $10.00[10.0-13.2]$ & $13.00[11.0-18.5]$ & 0.032 \\
FSS alta & $18.00[16.5-19.5]$ & $26.50[24.2-31.2]$ & 0.014 \\
Tempo de VMI (horas) & $144.0[102.0-228.0]$ & $72.00[13.0156 .0]$ & 0.053 \\
Tempo de internação UTI & $18.50[13.0-23.7]$ & $21.50[13.0-24.5]$ & 0.983 \\
\hline $\begin{array}{l}\text { Legenda: Teste Mann-Whitney; } \\
\text { invasiva; } \text { FSS- Functional Status Score Intervalo interquartil; VMI= ventilação }\end{array}$ & mecânica \\
\end{tabular}

Não houve diferença entre indivíduos sedados e não sedados na admissão em relação ao tempo de VMI $(p=0,504)$.

\section{DISCUSSÃO}

Os resultados deste estudo evidenciam correlação negativa entre o estado funcional da admissão e o tempo de VMI, onde quem apresentou pior estado funcional na admissão permaneceu por um tempo maior na VMI, bem como pacientes que ficaram por um período maior na VMI obtiveram piores resultados no estado funcional na alta. O presente estudo é pioneiro na avaliação do estado funcional numa amostra brasileira de pacientes em VMI e se insere na discussão multidimensional da funcionalidade, que é o principal domínio do fisioterapeuta.

Como forma de entendimento sobre a funcionalidade humana, a Classificação Internacional de Funcionalidade, Incapacidade e Saúde (CIF) é referência teórica norteadora em diversos contextos, inclusive no ambiente hospitalar, buscando entender a saúde e os estados de saúde, além das incapacidades dos indivíduos.

Sabe-se que restrição ao leito na UTI está associada a diversas condições como uso de dispositivos invasivos, sedativos, drogas vasoativas e até a própria $\mathrm{VMI}$, implicando em limitações para realização de atividades e restrições à participação social dos doentes. ${ }^{19,20}$

No presente estudo observou-se na avaliação funcional que houve uma diferença significativa em todos os domínios do FSS (A, B, C, D, E) e na pontuação total da admissão até a alta da UTI. 
Trush e colaboradores também encontraram melhora significativa em todas as 5 categorias do FSS da admissão até a alta, com melhores resultados nas categorias transferência de deitado para sentado e deambulação corroborando com os nossos achados. ${ }^{21}$

O que pode ter contribuído para esse resultado seria a realização de fisioterapia voltada para mobilização precoce. Sabe-se que a utilização de condutas com foco na mobilização precoce melhoram o desempenho físico dos pacientes, reduz tempo de desmame e otimiza a qualidade de vida pós alta. ${ }^{22}$

Schweickert et al. ${ }^{23}$ avaliaram a eficácia da mobilização precoce associada a interrupção diária da sedação em doentes críticos sob VMI e concluíram que a mobilização foi bem tolerada e segura, o que resultou em melhor estado funcional na alta e mais dias livres da VMI.

No presente estudo não houve correlação entre a morbimortalidade dos doentes com o tempo de VMI, possivelmente pela necessidade de estabilidade clínica dos pacientes que foi um prérequisito para avaliação funcional. Sabe-se que mobilização de doentes mecanicamente ventilados exige critérios de segurança já descritos na literatura. ${ }^{24}$

A utilização de fármacos vasoconstritores e vasodilatadores em alta vazão podem refletir a gravidade clínica dos pacientes neste ambiente específico. ${ }^{25}$ Neste estudo apenas $26 \%$ dos doentes utilizavam drogas vasoativas e em baixa vazão, o que confirma estabilidade clínica dos doentes e a sua não correlação com o tempo de VMI.

No presente estudo quando se compara os perfis diagnósticos, observa-se que os pacientes cirúrgicos obtiveram melhores resultados no que diz respeito ao tempo de VMI, tempo de internação hospitalar e estado funcional na alta em comparação com os doentes clínicos. Isso se deve, possivelmente, ao fato de que no pós-operatório das cirurgias abdominais os pacientes são liberados o quanto antes pela equipe médica para mobilização.

Já o doente clínico devido à condição inicial mais grave, geralmente evolui com complicações durante a internação e pior desfecho funcional na alta.

Jesus et al. ${ }^{6}$ divergem dos nossos achados, visto que avaliaram a capacidade funcional utilizando a Medida de Independência Funcional (MIF), numa amostra de pacientes predominantemente cirúrgicos e encontraram uma redução significativa da mobilidade (14,3\%) entre o momento admissão e a alta da UTI.

Em relação ao tempo de $\mathrm{VMI}$, não houve diferença entre indivíduos sedados e não sedados possivelmente pela necessidade de nível de consciência satisfatório para avaliação. Porém, sabe-se que a administração contínua de sedativos pode resultar em aumento do tempo de VMI e pior desfecho clinico. ${ }^{26}$

Shehabi et al. ${ }^{27}$ avaliaram a relação entre o uso de sedação, tempo de extubação, delirium e mortalidade em pacientes críticos na Austrália e nova Zelândia e concluíram que a sedação profunda é um preditor negativo independente para o atraso no tempo de extubação e aumento da mortalidade a longo prazo.

Em outro estudo semelhante Strom et al. ${ }^{28}$ também observaram um aumento significativo de dias sem uso de VMI no grupo não sedado quando comparado ao grupo com interrupção diária da sedação.

Foram limitações deste estudo a população heterogênea que incluiu pacientes clínicos e pós-cirúrgicos, o pequeno tamanho amostral devido ao curto período de tempo para coleta de dados e a não realização de avaliação funcional prévia ao internamento, limitando dessa forma uma análise estatística mais detalhada. Sugerese novos estudos com acompanhamento dos doentes críticos após a alta da UTI para quantificação dos desfechos e sua correlação com o estado funcional.

\section{CONCLUSÃO}

Existe correlação negativa entre a evolução do estado funcional na admissão e alta com o tempo de VMI em doentes críticos. O perfil diagnóstico também pode interferir no estado funcional, uma vez que pacientes cirúrgicos apresentam melhores resultados em relação aos pacientes clínicos. Contudo, não foi possível identificar no presente estudo os fatores etiológicos e prognósticos que influenciaram nesse desfecho e, portanto, faz-se necessário, a realização de mais estudos com este fim.

\section{REFERÊNCIAS}

1. Mondadori AG, Zeni EM, Oliveira A, Silva CC, Wolf VLW, Taglietti M. Humanização da fisioterapia em Unidade de Terapia Intensiva Adulto: estudo transversal. Fisioter Pesq. 2016;23(3):294-300. Doi: http://dx.doi.org/10.1590/1809-2950/16003123032016

2. Reis LCC, Gabarra LM, Moré CLOO. As repercussões do processo de internação em UTI adulto na perspectiva de familiares. Temas Psicol. 2016;24(3):815-28.

Doi: http://dx.doi.org/10.9788/TP2016.3-03

3. Santos LJ, Silveira FS, Muller FF, Araújo HD, Comerlato JB, Silva MC, et al. Avaliação funcional de pacientes internados na unidade de terapia intensiva adulto do Hospital Universitário de Canoas. Fisioter Pesq. 2017;24(4):437-43. Doi: https://doi.org/10.1590/1809-2950/17720924042017

4. Silva FRR, Souza TB, Dias MS, Silva APP, Oliveira KC, Oliveira MML, et al. Avaliação da capacidade funcional dos pacientes em uso de ventilação mecânica internados em uma Unidade de Terapia Intensiva. Rev HUPE. 2017;16(1):6-15. Doi: http://dx.doi.org/10.12957/rhupe.2017.33299

5. Ferreira NA, Lopes AJ, Ferreira AS, Ntoumenopoulos G, Dias J, Guimaraes FS. Determination of functional prognosis in hospitalized patients following an intensive care admission. World J Crit Care Med. 2016;5(4):219-227. Doi: http://dx.doi.org/10.5492/wjccm.v5.i4.219

6. Jesus FS, Paim DM, Brito JO, Barros IA, Nogueira TB, Martinez BP, et al. Mobility decline in patients hospitalized in an intensive care unit. Rev Bras Ter Intensiva. 2016;28(2):114-9. Doi: http://dx.doi.org/10.5935/0103-507X.20160025

7. Curzel J, Forgiarini Júnior LA, Rieder MM. Avaliação da independência funcional após alta da unidade de terapia intensiva. Rev Bras Ter Intensiva. 2013;25(2):93-8. Doi: https://doi.org/10.5935/0103-507X.20130019

8. Martinez BP, Bispo AO, Duarte ACM, Gomes Neto M. Declínio funcional em uma unidade de terapia intensiva (UTI). Rev Inspirar Mov Saúde. 2013;5(1):1-5.

9. Gomes RHS, Santos RS. Avaliação da capacidade e comprometimento funcional em pacientes traqueotomizados de um hospital público de Curitiba. Rev CEFAC. 2016;18(1):120-8. Doi: https://doi.org/10.1590/1982-021620161813715

10. Cordeiro AL, Melo TA, Santos AM, Lopes GF. Influência do tempo de ventilação mecânica sobre a independência funcional em pacientes submetidos à cirurgia cardíaca: uma revisão da literatura. Fisioter Mov. 2015;28(4):859-64. Doi: http://dx.doi.org/10.1590/0103-5150.028.004.AR04

11. Costa FM, Correa ADB, Narala Neto E, Vieira EMM, Lima E, Nasrala $M L S$, et al. Avaliação da funcionalidade motora em pacientes com tempo prolongado de internação hospitalar. UNOPAR Cient Ciênc Biol Saúde. 2014;16(2):87-91. Doi: https://doi.org/10.17921/2447-8938.2014v16n2p\%25p

12. Cordeiro ALL, Brito AAOR, Santana NMA, Silva INMS, Nogueira SCO, Guimarães ARF, et al. Análise do grau de independência funcional pré e na alta da Unidade de Terapia Intensiva em pacientes submetidos à cirurgia cardíaca. Rev Pesq Fisioter. 2015; 5(1):21-27. Doi: http://dx.doi.org/10.17267/22382704rpf.v5i1.574

13. Calvo-Ayala E, Khan BA, Farber MO, Ely EW, Boustani MA. Interventions to improve the physical function of ICU survivors: a systematic review. Chest. 2013;144(5):1469-1480. Doi: http://dx.doi.org/10.1378/chest.13-0779 
14. Silva VZM, Araujo Neto JA, Cipriano Junior G, Pinedo M, Needham DM, Zanni JM, et al. Versão brasileira da Escala de Estado Funcional em UTI: tradução e adaptação transcultural. Rev Bras Ter Intensiva. 2017;29(1):34-8. Doi: http://dx.doi.org/10.5935/0103-507X.20170006

15. Christakou A, Papadopoulos E, Patsaki I, Giorgos S, Serafeim N. Functional Assessment Scales in a General Intensive Care Unit. A review. Hospital Chronicles. 2013;8(4):164-70. Doi: https://doi.org/10.2015/hc.v8i4.552

16. Charlson ME, Pompei P, Ales KL, MacKenzie CR. A new method of classifying prognostic comorbidity in longitudinal studies: development and validation. J Chronic Dis. 1987;40(5):373-83. Doi: http://dx.doi.org/10.1016/0021-9681(87)90171-8

17. lucif JRN, Rocha JSY. Estudo da desigualdade na mortalidade hospitalar pelo índice de comorbidades de Charlson. Rev Saúde Pública. 2004;38(6):780-6. Doi: https://doi.org/10.1590/S003489102004000600005

18. Portney LG, Watkins MP. Foundations of clinical research applications to pratice. Connecticut; Appleton\& Lange; 1997.

19. CIF: Classificação Internacional de Funcionalidade, Incapacidade e Saúde. São Paulo: Edusp; 2003.

20. Levack K. The International Classification of Functioning, Disability and Health (ICF): applications to physiotherapy. NZJP. 2004;32:12.

21. Thrush A, Rozek M, Dekerlegand JL. The clinical utility of the functional status score for the intensive care unit (FSS-ICU) at a long-term acute care hospital: a prospective cohort study. Phys Ther. 2012;92(12):1536-45

Doi: http://dx.doi.org/10.2522/ptj.20110412
22. Hodgson C, Bellomo R, Berney S, Bailey M, Buhr H, Denehy L, et al. Early mobilization and recovery in mechanically ventilated patients in the ICU: a bi-national, multi-centre, prospective cohort study. Crit Care. 2015;19(1):81. Doi: http://dx.doi.org/10.1186/s13054-015-0765-4

23. Schweickert WD, Pohlman MC, Pohlman AS, Nigos C, Pawlik AJ, Esbrook $\mathrm{CL}$, et al. Early physical and occupational therapy in mechanically ventilated, critically ill patients: a randomised controlled trial. Lancet. 2009;373(9678):1874-82. Doi: http://dx.doi.org/10.1016/S0140-6736(09)60658-9

24. Hodgson CL, Stiller $K$, Needham DM, Tipping CJ, Harrold $M$, Baldwin CE, et al. Expert consensus and recommendations on safety criteria for active mobilization of mechanically ventilated critically ill adults. Crit Care. 2014;18(6):658. Doi: http://dx.doi.org/10.1186/s13054-014-0658-y

25. Fonseca L, Vieira FN, Azzolin KO. Fatores associados ao tempo de ventilação mecânica no pós-operatório de cirurgia cardíaca. Rev Gaucha Enferm. 2014;35(2):67-72. Doi: http://dx.doi.org/10.1590/1983-1447.2014.02.44697

26. Braganca HCE, Rezende AAB, Reis GR, Magagnin JLS, Miranda EF, Rodrigues ESR, et al. Correlação entre níveis de sedação e tempo de ventilação mecânica. Rev Amazônia Science \& Health. 2014;2(2):15-20.

27. Shehabi Y, Bellomo R, Reade MC, et al. Early intensive care sedation predicts long-term mortality in ventilated critically ill patients. Am J Respir Crit Care Med. 2012;186(8):724-731. Doi: http://dx.doi.org/10.1164/rccm.201203-05220C

28. Strøm T, Martinussen T, Toft P. A protocol of no sedation for critically ill patients receiving mechanical ventilation: a randomised trial. Lancet. 2010;375(9713):475-80. Doi: http://dx.doi.org/10.1016/S0140-6736(09)62072-9 